\title{
BETTER FINGERPRINT IMAGE COMPRESSION AT LOWER BIT-RATES: AN APPROACH USING MULTIWAVELETS WITH OPTIMISED PREFILTER COEFFICIENTS
}

\author{
Rema N.R ${ }^{1}$, Shanavaz K.T ${ }^{2}$ and Mythili $\mathbf{P}^{3}$ \\ ${ }^{1,3}$ Department of Electronics and Communication Engineering, School of Engineering, Cochin University of Science \& Technology, India \\ ${ }^{2}$ Department of Electronics and Communication Engineering, College of Engineering, Kallooppara, India
}

\begin{abstract}
In this paper, a multiwavelet based fingerprint compression technique using set partitioning in hierarchical trees (SPIHT) algorithm with optimised prefilter coefficients is proposed. While wavelet based progressive compression techniques give a blurred image at lower bit rates due to lack of high frequency information, multiwavelets can be used efficiently to represent high frequency information. SA4 (Symmetric Antisymmetric) multiwavelet when combined with SPIHT reduces the number of nodes during initialization to $1 / 4^{\text {th }}$ compared to SPIHT with wavelet. This reduction in nodes leads to improvement in PSNR at lower bit rates. The PSNR can be further improved by optimizing the prefilter coefficients. In this work genetic algorithm $(G A)$ is used for optimizing prefilter coefficients. Using the proposed technique, there is a considerable improvement in PSNR at lower bit rates, compared to existing techniques in literature. An overall average improvement of $4.23 \mathrm{~dB}$ and $2.52 \mathrm{~dB}$ for bit rates in between 0.01 to 1 has been achieved for the images in the databases FVC $2000 \mathrm{DB} 1$ and FVC 2002 DB3 respectively. The quality of the reconstructed image is better even at higher compression ratios like 80:1 and 100:1. The level of decomposition required for a multiwavelet is lesser compared to a wavelet.
\end{abstract}

Keywords:

Multiwavelet, Fingerprint, Compression, Lower Bit Rate, Optimised Prefilter Coefficients

\section{INTRODUCTION}

The fingerprint is one of the most widely used biometrics used for identification of an individual in the areas of law enforcement and immigration. For the transmission and storage of a large number of fingerprints, compression is essential. Compression is of two types, lossy compression and lossless compression. With lossless methods, a compression ratio of $2: 1$ or $3: 1$ can be achieved. But if some amount of information loss can be tolerated, a compression ratio of $15: 1$ or more can be achieved by lossy compression. Fingerprint compression comes under lossy method. FBI (Federal Bureau of Investigation) uses Wavelet Scalar Quantization (WSQ) as the compression standard for the storage of fingerprint images. Wavelet based compression algorithms are generally preferred over standard compression algorithms since WSQ prevents blocking artifacts at higher compression ratios.

Multiwavelets are a generalized form of wavelet and can be used as a transform in image compression instead of the wavelet. Strela et al. [1] showed that multiwavelet with the appropriate combination of prefilter will be an efficient substitute for scalar wavelet. Ashok and Reddy [2] proposed a compression technique based on multiwavelet transform for natural images. They proved that multiwavelets can excel at preserving high frequency components in images.

Ragupathy et al. [3] applied multiwavelet based SPIHT algorithm for the compression of images without shuffling the detail coefficients and with shuffling in the approximation coefficients. In this, the number of decomposition levels in multiwavelet was taken as maximum, not an optimum value. Radhakrishnan and Subramaniam [4] proposed fingerprint compression with modifications in the scanning order of multiwavelet coefficients in SPIHT algorithm without performing coefficient shuffling. In addition to this, all the coefficients in sixteen frequency subbands were used for initialization. Hence the PSNR at low bit rates become degraded and it was not taken into consideration. No standard database was used for validation.

Unlike wavelets, multiwavelets require prefiltering for applying the decomposition filters [5]. The performance of multiwavelet can be improved by using suitable prefilters. Attakitmongcol et al. [6] proposed optimum prefilters for DGHM (Donovan Geronimo Hardin Massopust) and CL (Chui-Lian) multiwavelets. A better performance in compression compared to Daubechies-4 wavelet and a poor performance compared to bior 4.4 wavelet was reported. Shi et al. [7] proposed a new prefilter design by maximizing the proportion of frequency spectrum energy of low-pass filters in low-frequency band to total frequency spectrum energy. In their paper, prefilter was designed on the assumption that high frequencies contain unimportant information and low frequencies contain important information.

At low bit rates traditional compression techniques are not effective in reconstructing a good quality image. Esakkirajan et al. [8] proposed a fingerprint compression technique based on contourlet and multistage vector quantization. An improvement in performance upto 0.125 bits per dimension was reported at the expense of execution time. Shao et al. [9] proposed a sparse representation based fingerprint compression technique. The maximum compression ratio considered was 40:1 and the algorithm exhibited higher complexity due to block processing. For the compression of fingerprint images, Shanavaz and Mythili [10] proposed evolution of wavelet lifting coefficients and produced an improvement of $1.009 \mathrm{~dB}$ in average PSNR. They reported the performance of compression at very low bit rates like 0.01 which are not satisfactory at lower levels of decomposition. Emmanuel et al. [11] proposed fingerprint compression using discrete wavelet transform, Lloyd-Max non-uniform quantization and entropy coding. They obtained an improvement in terms of compression ratio 20:1 over existing algorithms which achieved a compression ratio of 15:1. A compression scheme for set partitioning coders for improving the verification performance of 
fingerprints at low bit rates was proposed by Grailu [12]. In this the local variations in the compressed image were minimized.

To overcome the low bit rate issues, a multiwavelet based fingerprint image compression technique using SPIHT with optimised prefilter coefficients is proposed in this paper. The multiwavelet used here is SA4. The multiwavelet without coefficient shuffling has been used to effectively utilize its decomposition structure. An improved PSNR at all bit rates is achieved using this method.

\section{FINGERPRINT COMPRESSION USING MULTIWAVELETS}

In wavelet based progressive compression techniques, the compressed image becomes degraded due to blurring and ringing artifacts at low bit rates. This is due to the ineffectiveness of wavelets in representing high frequency information. Fingerprint contains a lot of high frequency information and SPIHT algorithm fails to capture them at lower bit rates. For a given level of decomposition multiwavelets have more frequency information compared to wavelets which can be effectively utilized to get a better reconstructed image. Hence multiwavelets can be better candidates for image compression. The Fig. 1 shows the general block diagram of a lossy fingerprint compression using multiwavelets. The fingerprint image is prefiltered to rearrange the data suitable for applying multiwavelet transform. After transforming this data using multiwavelet, the transformed image is given as input to the SPIHT algorithm to get a compressed bit stream. This compressed bit stream when applied with inverse operations (inverse SPIHT, inverse transform, optimum post filter) gives back the reconstructed image.

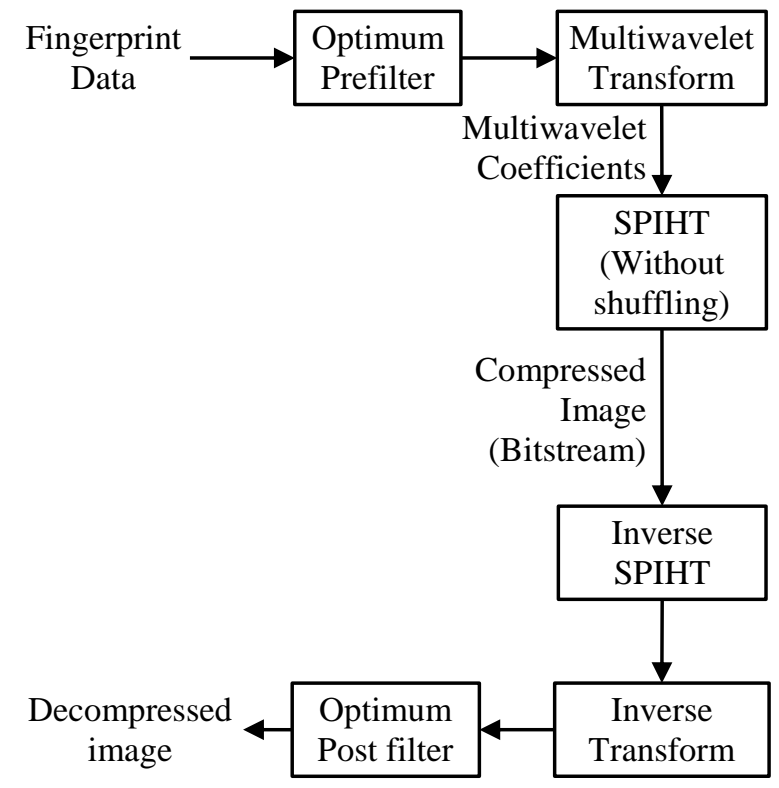

Fig.1. Block diagram of fingerprint compression

SPIHT is a wavelet based compression algorithm which works by exploiting the similarities of wavelet transform coefficients in different decomposition levels. This type of similarity does not exist in multiwavelets. To get a decomposition structure similar to wavelet, Martin and Bell [13] proposed coefficient shuffling for multiwavelets. Due to shuffling, the number of nodes remained the same for both wavelet and multiwavelet in the SPIHT initialization process. The performance of SPIHT at low bit rates is inversely proportional to the number of nodes. Hence to improve the performance at low bit rates, the number of nodes should be reduced. This can be achieved by maintaining the coefficients unshuffled. If the number of nodes required for initialization in SPIHT using a shuffled multiwavelet is $M$, the number of nodes reduces to $\mathrm{M} / 4$ for an unshuffled multiwavelet.

\subsection{MULTIWAVELET TRANSFORM}

The standard wavelet based multiresolution analysis (MRA) has one scaling function $\Phi(t)$ and one wavelet function $\Psi(t)$. Similar to scalar wavelets, the theory of multiwavelets is based on MRA. For multiwavelets, the dilation Eq.is given by Eq.(1)

$$
\Phi(t)=\sum_{n} H(n) \Phi(2 t-n)
$$

where, $\Phi(t)=\left[\Phi_{1}(t), \ldots, \Phi_{N}(t)\right]^{T}$ with $N$ scaling functions and $H(n)$ is the scaling filter of dimension $N \times N$. The wavelet is given by Eq.(2),

$$
\omega(t)=\sum_{n} G(n) \Phi(2 t-n)
$$

where, $\omega(t)=\left[\omega_{1}(t), \ldots, \omega_{N}(t)\right]^{T}$ with $N$ wavelet functions and $G(n)$ is the wavelet filter of dimension $N \times N$. While multiwavelets can possess orthogonality, symmetry, compact support and a large number of vanishing moments simultaneously, scalar wavelet cannot possess these properties simultaneously. Sumalatha and Subramanyam [14] compared the performance of different multiwavelets in compression of medical images and showed that SA4 multiwavelet gives the best performance.

\subsection{SA4 MULTIWAVELET}

Multiwavelet proposed by Tham et al. [15] was designed to be satisfying good multifilter properties (GMPs). They showed that SA4 multiwavelet possessing GMP can perform well in compression applications compared to other multiwavelets which do not possess GMP. SA4 belong to a family of multiwavelet filters in which scaling and wavelet functions are symmetric and antisymmetric pairs. The low pass filter coefficients are given by Eq.(3),

$$
\begin{gathered}
H_{0}=\frac{1}{\sqrt{2}} \frac{1}{v^{2}+1}\left[\begin{array}{cc}
1 & v \\
1 & -v
\end{array}\right], \\
H_{0}=\frac{1}{\sqrt{2}} \frac{1}{v^{2}+1}\left[\begin{array}{cc}
v^{2} & v \\
-v^{2} & v
\end{array}\right], \\
H_{2}=S H_{1} S, \\
H_{3}=S H_{0} S
\end{gathered}
$$

and the high pass filter coefficients are given by Eq.(4).

$$
\begin{gathered}
G_{0}=\frac{1}{\sqrt{2}} \frac{1}{v^{2}+1}\left[\begin{array}{cc}
v & -1 \\
v & 1
\end{array}\right], \\
G_{1}=\frac{1}{\sqrt{2}} \frac{1}{v^{2}+1}\left[\begin{array}{cc}
-v & v^{2} \\
v & v^{2}
\end{array}\right], \\
G_{2}=S G_{1} S,
\end{gathered}
$$




$$
G_{3}=S G_{0} S
$$

where, $S=\operatorname{diag}(1,-1)$ and $v=4+\sqrt{15}$

Generally for multiwavelets scaling and wavelet filter coefficients are $2 \times 2$ matrices. So for applying multiwavelets a single row of input should be converted into 2 rows. This can be done by either oversampling or critical sampling the input. Oversampling performed by repeating the input row is not suitable for compression since it introduces redundancies. Critical sampling which is usually used for compression, is performed by dividing a single row of input into odd and even components so that a single $1 \times \mathrm{N}$ row can be converted into a matrix of size $2 \times N / 2$. Dividing into odd and even components can change the characteristics of the input signal. To preserve the characteristics of the input signal, the $2 \times N / 2$ input matrix is pre-multiplied by a prefilter.

\subsection{OPTIMISATION COEFFICIENTS \\ OF PREFILTER}

Prefilter coefficients have an important role in arranging the information in different frequency bands which can be utilized for compression. The performance of multiwavelet is strongly dependent on the selection of prefilters. In [15] an orthogonal, low complexity prefilter which works well with any multiwavelet was proposed. The prefilter design of SA4 multiwavelet was based on the assumption that the elements of input signal were locally smooth and the prefilter coefficients are given by Eq.(5),

$$
P R=\frac{1}{\sqrt{2}}\left[\begin{array}{cc}
1 & 1 \\
-1 & 1
\end{array}\right] .
$$

Prefilters available in the literature were on the assumption that low frequencies contain important information. But in the case of fingerprint images, it can be observed that both high as well as low frequencies contain important information. Hence traditional prefilters are not suitable for fingerprint image compression and there is a need for optimum prefilter which can represent both high and low frequencies equally well. In this paper, GA has been used for optimizing prefilter coefficients of the SA4 multiwavelet. The energy in different frequency bands of the SA4 multiwavelet changes with varying prefilters. Any multiwavelet with different prefilters changes the PSNR of compressed image. The performance of multiwavelet with appropriate prefilters gives better results in compression performance.

\subsection{SPIHT FOR MULTIWAVELETS}

SPIHT proposed by Said and Pearlman [16] is a progressive transmission technique so that at any moment the quality of the image is the best available for the received bits up to that moment. In this algorithm, the wavelet coefficients to be transmitted are stored in 2 lists; List of insignificant pixels (LIP) and List of insignificant sets (LIS). During initialization, these lists are initialized by coefficients in the lowest frequency subband (LL band) of the transform. A threshold is set based on the maximum magnitude of the transform coefficient and the threshold is reduced in subsequent passes. The nodes in LIP and then in LIS are tested for significance and the resulting bits are transmitted. A group of transform coefficients is represented by a set in LIS and a single bit is transmitted for the significance of the entire set.
For a single level decomposition, a scalar wavelet has four frequency subbands whereas a multiwavelet has sixteen frequency subbands as shown in Fig.2(a) and Fig.2(b). There are four frequency bands in the approximation and detail subbands creating a total of sixteen subbands. Multiwavelet after coefficient shuffling as shown in Fig.2(c) has a decomposition structure similar to wavelet and these shuffled coefficients were generally used for compression. In this paper an investigation in to using the unshuffled coefficients as such has been carried out. The lowest frequency subband (LL band) of the unshuffled multiwavelet is $1 / 4^{\text {th }}$ the size of the lowest frequency subband of shuffled multiwavelet. As LIP and LIS are initialized with the nodes in lowest frequency subband of multiwavelet transform, the number of nodes in LIP and LIS can be reduced to $1 / 4^{\text {th }}$ using the unshuffled multiwavelet. In SPIHT algorithm, the initial threshold will be a high value and most of the transform coefficients will be insignificant at low bit rates. If the number of nodes presents in LIP and LIS are less, the number of insignificant bits transmitted can be reduced. So more amount of significant information can be included in the bit stream and a better performance can be achieved at low bit rates. This confirms the effectiveness of retaining the coefficients unshuffled.

SPIHT algorithm contains three steps, initialization, sorting pass and refinement pass. During initialization step of SPIHT algorithm, LIP and LIS are initialized with lowest frequency coefficients in the highest level of decomposition of SA4 multiwavelet. When SA4 multiwavelet is applied to SPIHT algorithm, LIP and LIS are initialized with coefficients in $\mathrm{L}_{1} \mathrm{~L}_{1}$ band of multiwavelet (Fig.2(b)) instead of LL band of shuffled multiwavelet (Fig.2(c)). During sorting pass, coefficient in LIP and then in LIS are tested for significance and the remaining steps of sorting and refinement passes are the same as that of SPIHT with wavelets.

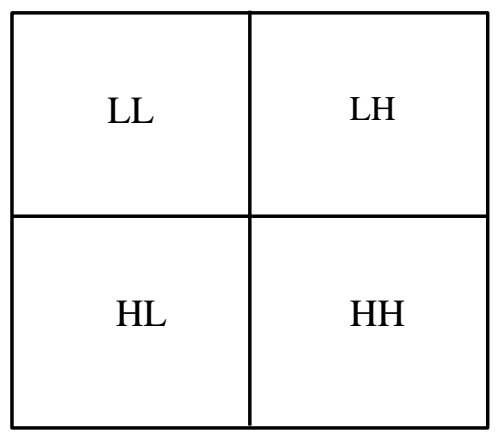

\begin{tabular}{|c|c|c|c|}
\hline \begin{tabular}{l|l|l}
15 \\
$L_{1} L_{1}$
\end{tabular} & \begin{tabular}{|c|c|}
2 & 6 \\
$L_{1} L_{2}$
\end{tabular} & $\mathrm{~L}_{1} \mathrm{H}_{1}$ & $\mathrm{~L}_{1} \mathrm{H}_{2}$ \\
\hline \begin{tabular}{l|l|}
3 & 7 \\
& $L_{2} L_{1}$
\end{tabular} & \begin{tabular}{|l|l|l|}
4 & 8 \\
& $L_{2} L_{2}$
\end{tabular} & $\mathrm{~L}_{2} \mathrm{H}_{1}$ & $\mathrm{~L}_{2} \mathrm{H}_{2}$ \\
\hline $\mathrm{H}_{1} \mathrm{~L}_{1}$ & $\mathrm{H}_{1} \mathrm{~L}_{2}$ & $\mathrm{H}_{1} \mathrm{H}_{1}$ & $\mathrm{H}_{1} \mathrm{H}_{2}$ \\
\hline $\mathrm{H}_{2} \mathrm{~L}_{1}$ & $\mathrm{H}_{2} \mathrm{~L}_{2}$ & $\mathrm{H}_{2} \mathrm{H}_{1}$ & $\mathrm{H}_{2} \mathrm{H}_{2}$ \\
\hline
\end{tabular}

(a)

(b) 


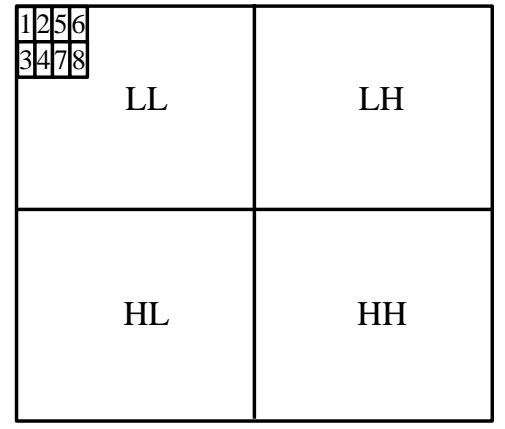

(c)

Fig.2. Single level decomposition of (a) Scalar wavelet

(b) Multiwavelet (c) Multiwavelet with shuffling

\section{DATABASE \\ AND \\ PERFORMANCE MEASURES}

Fingerprint verification competition (FVC) 2000 DB1 and FVC 2002 DB3 are the databases used for validating the proposed technique. Each of these databases contains 80 fingerprint images. The size of image is $300 \times 300$ pixels and with a resolution of 500 dpi. Center cropped images with size as $256 \times 256$ are taken as the input. For measuring the quality of compression, Peak Signal to Noise Ratio (PSNR) is used and is given by Eq.(6),

$$
P S N R=20 \log _{10} \frac{255}{R M S E}
$$

where RMSE is the root mean square error between original and reconstructed image.

Quantitative measure used for measuring the amount of compression achieved is given by compression ratio (CR)

$$
C R=\frac{\text { Size of input image }}{\text { Size of output image }} \text {. }
$$

Higher the value of compression ratio, more the amount of compression achieved.

\section{RESULTS AND DISCUSSIONS}

Initially the fingerprint image is transformed into multiwavelet domain. The multiwavelet used is SA4 with existing prefilter. The input fingerprint image is shown in Fig.3(a) and multiwavelet transformed image with four level of decomposition is shown in Fig.3(b). Further these multiwavelet coefficients are applied to the SPIHT compression algorithm

Before validating and analyzing the proposed algorithm, the impact on PSNR with varying the level of transform was studied. It is known that the performance of wavelet is varying for different decomposition levels. When the number of decomposition levels increases, the performance of compression increases first, reaching a maximum value and then remains almost constant. As the number of decomposition levels increases, the number of bits required to represent transform coefficient increases. Further the boundary effects associated with filtering can reduce the coding efficiency [17]. So there is a limit to the number of decomposition levels while using the transform.
The decomposition levels can be increased until the size of the last decomposed subband becomes equal to the filter length.

The performance of unshuffled SA4 multiwavelet with existing prefilter is compared with that of the wavelet [10] with reference to the level of decomposition. Fingerprint images from FVC 2000 DB1 were considered for comparing the performance and the average PSNR of 80 images for bit rates 0.01 to 1 were computed. The performance of the wavelet and multiwavelet with existing prefilter coefficients for different levels of decomposition is shown in Table.1. From the Table.1 it can be seen that compression using multiwavelet with 4 level of decomposition is giving a performance better than wavelet with 5 level decomposition at lower bit rate.

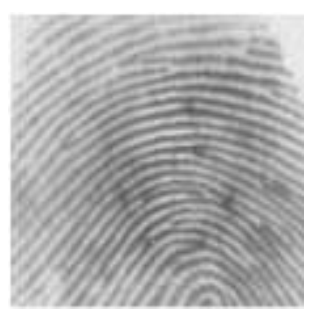

(a)

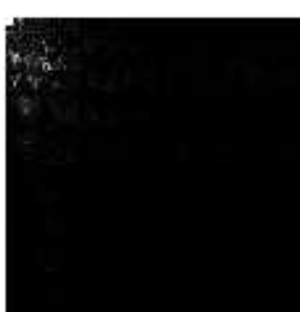

(b)
Fig.3. (a) Input image (b) Multiwavelet transform

\subsection{PERFORMANCE OF PROPOSED METHOD}

To validate and analyze the proposed algorithm, 80 images from FVC 2000 DB1 and FVC 2002 DB3 database were considered. The performance of the proposed technique is compared initially with that of the optimised wavelet proposed by [10]. For the images in FVC 2000 DB1 database the average PSNR obtained by the authors, varied from $7.536 \mathrm{~dB}$ to $40.566 \mathrm{~dB}$ for bit rates ranging from 0.01 to 1 bits per pixel (bpp) as shown in column 2 of Table.2. The level of decomposition was taken as 4 for both the wavelet and multiwavelet for fair comparison purpose. Next the average PSNR for the same database was computed with and without shuffling the SA4 multiwavelet with unoptimised prefilter coefficients (Eq.(5)) and is shown in columns 3 and 4 of Table.2. In the case of SA4 multiwavelet with coefficient shuffling, the average PSNR varied from $18.047 \mathrm{~dB}$ to $38.631 \mathrm{~dB}$. The improvement in PSNR was limited up to $0.1 \mathrm{bpp}$. In order to improve the performance over the other bit rates, SA4 multiwavelet without coefficient shuffling was tried. This provided an overall average improvement of 5.59dB in PSNR up to $0.5 \mathrm{bpp}$. It can be seen from the Table. 2 that, the multiwavelet with unshuffled coefficients performed better compared to multiwavelet with shuffled coefficients and wavelets at low bit rates. This might be due to the lesser number of nodes used for initialization of lists in SPIHT algorithm. The performance of the multiwavelet can be further improved by optimizing the prefilter coefficients.

SA4 prefilter coefficients were earlier optimised by Tham et al. [13] on the assumption that adjacent coefficients are approximately equal. Further improvement in performance might be achieved by optimizing the prefilter coefficients based on the conditions mentioned in section 2.3. Here in this work, SA4 prefilter coefficients are optimised using GA for maximizing the PSNR. The optimised prefilter coefficients obtained using GA in matrix form is given by Eq.(8), 


$$
P R_{o}=\left[\begin{array}{cc}
0.9931 & 0.9980 \\
-0.7951 & 0.7961
\end{array}\right]
$$

The PSNR computed using the optimum SA4 prefilter coefficients are given in column 5 of Table.2. With the optimised prefilter, the average PSNR varies from $18.498 \mathrm{~dB}$ to $40.618 \mathrm{~dB}$ for bit rates 0.01 to 1 . Similarly for the images in FVC 2002 DB3 database the PSNR varied from $12.208 \mathrm{~dB}$ to $30.860 \mathrm{~dB}$ for the case of wavelets and from $17.990 \mathrm{~dB}$ to $31.136 \mathrm{~dB}$ for multiwavelets with optimum prefilter coefficients. Here again the overall performance has improved with unshuffled multiwavelet with optimum prefilter. An overall average improvement of $4.23 \mathrm{~dB}$ for the 80 images in FVC $2000 \mathrm{DB} 1$ database and $2.52 \mathrm{~dB}$ for the images in FVC 2002 DB3 database has been achieved using the proposed technique.

Table.1. Average PSNR of images in FVC 2000 DB1 database for different levels of decomposition

\begin{tabular}{|c|c|c|c|c|c|c|c|c|c|}
\hline \multirow{2}{*}{ bpp } & \multirow{2}{*}{ CR } & \multicolumn{4}{|c|}{ Wavelet [10] } & \multicolumn{4}{|c|}{$\begin{array}{c}\text { Unshuffled multiwavelet with } \\
\text { existing prefilter }\end{array}$} \\
\hline & & $\begin{array}{c}\text { Level } \\
3\end{array}$ & $\begin{array}{c}\text { Level } \\
4\end{array}$ & $\begin{array}{l}\text { Level } \\
5\end{array}$ & $\begin{array}{l}\text { Level } \\
6\end{array}$ & $\begin{array}{c}\text { Level } \\
3\end{array}$ & $\begin{array}{c}\text { Level } \\
4\end{array}$ & $\begin{array}{c}\text { Level } \\
5\end{array}$ & $\begin{array}{c}\text { Level } \\
6\end{array}$ \\
\hline 0.01 & 819.2 & 3.344 & 7.536 & 18.317 & 22.862 & 9.149 & 18.471 & 23.243 & 23.781 \\
\hline 0.03 & 268.5 & 5.060 & 15.522 & 22.906 & 24.578 & 17.984 & 23.175 & 24.704 & 25.110 \\
\hline 0.05 & 160.7 & 9.848 & 17.121 & 24.062 & 25.875 & 18.520 & 24.441 & 25.931 & 26.263 \\
\hline 0.07 & 114.6 & 9.848 & 18.459 & 25.361 & 26.971 & 20.539 & 25.784 & 26.936 & 27.209 \\
\hline 0.09 & 89.1 & 9.848 & 21.422 & 26.551 & 27.960 & 22.528 & 26.821 & 27.807 & 28.059 \\
\hline 0.2 & 40.0 & 13.573 & 26.541 & 31.095 & 31.960 & 27.793 & 30.772 & 31.335 & 31.476 \\
\hline 0.4 & 20.0 & 21.620 & 33.596 & 35.836 & 36.271 & 33.318 & 34.660 & 34.948 & 35.024 \\
\hline 0.6 & 13.3 & 27.234 & 37.175 & 38.396 & 38.641 & 36.029 & 36.972 & 37.182 & 37.235 \\
\hline 0.8 & 10 & 31.985 & 39.152 & 39.947 & 40.139 & 37.944 & 38.619 & 38.783 & 38.827 \\
\hline 1 & 8 & 35.905 & 40.566 & 41.214 & 41.364 & 39.428 & 40.034 & 40.185 & 40.224 \\
\hline
\end{tabular}

Table.2. Average PSNR in dB for images in FVC 2000 DB1 and FVC 2002 DB3 databases

\begin{tabular}{|c|c|c|c|c|c|c|c|c|}
\hline \multirow{2}{*}{ bpp } & \multicolumn{4}{|c|}{ FVC 2000 DB1 } & \multicolumn{4}{c|}{ FVC 2002 DB3 } \\
\cline { 2 - 8 } & $\begin{array}{c}\text { Wavelet } \\
{[\mathbf{1 0}]}\end{array}$ & $\begin{array}{c}\text { Sa4 shuffled } \\
{[\mathbf{1 3}]}\end{array}$ & $\begin{array}{c}\text { Sa4 } \\
\text { unshuffled }\end{array}$ & $\begin{array}{c}\text { Sa4 optimal } \\
\text { pre-filtering }\end{array}$ & $\begin{array}{c}\text { Wavelet } \\
{[\mathbf{1 0}]}\end{array}$ & $\begin{array}{c}\text { Sa4 shuffled } \\
{[\mathbf{1 3}]}\end{array}$ & $\begin{array}{c}\text { Sa4 } \\
\text { unshuffled }\end{array}$ & $\begin{array}{c}\text { Sa4 optimal } \\
\text { pre-filtering }\end{array}$ \\
\hline 0.01 & 7.536 & 18.047 & 18.471 & 18.498 & 12.208 & 11.159 & 17.915 & 17.990 \\
\hline 0.02 & 15.519 & 18.047 & 20.293 & 20.222 & 13.276 & 11.159 & 19.979 & 19.982 \\
\hline 0.03 & 15.522 & 18.308 & 23.175 & 23.159 & 13.539 & 13.213 & 21.924 & 21.928 \\
\hline 0.04 & 17.121 & 18.471 & 23.861 & 23.860 & 18.811 & 17.915 & 22.345 & 22.339 \\
\hline 0.05 & 17.121 & 18.471 & 24.441 & 24.433 & 18.811 & 17.915 & 22.670 & 22.674 \\
\hline 0.06 & 18.459 & 20.293 & 25.150 & 25.154 & 20.058 & 19.979 & 23.100 & 23.118 \\
\hline 0.07 & 18.459 & 20.293 & 25.784 & 25.798 & 20.375 & 19.979 & 23.463 & 23.476 \\
\hline 0.08 & 18.459 & 20.293 & 26.330 & 26.344 & 20.375 & 19.979 & 23.742 & 23.765 \\
\hline 0.09 & 21.422 & 22.166 & 26.821 & 26.832 & 21.590 & 21.350 & 23.989 & 24.008 \\
\hline 0.1 & 21.422 & 22.167 & 27.265 & 27.297 & 21.596 & 21.358 & 24.210 & 24.234 \\
\hline 0.2 & 26.541 & 26.467 & 30.772 & 30.894 & 24.393 & 23.902 & 25.838 & 25.913 \\
\hline 0.3 & 30.726 & 30.009 & 33.050 & 33.316 & 26.070 & 25.483 & 26.786 & 26.911 \\
\hline 0.4 & 33.596 & 32.300 & 34.660 & 35.061 & 27.125 & 26.402 & 27.550 & 27.703 \\
\hline 0.5 & 35.663 & 34.065 & 35.880 & 36.443 & 27.879 & 27.054 & 28.250 & 28.362 \\
\hline 0.6 & 37.175 & 35.230 & 36.972 & 37.566 & 28.594 & 27.704 & 28.878 & 28.956 \\
\hline 0.7 & 38.316 & 36.314 & 37.840 & 38.464 & 29.198 & 28.315 & 29.433 & 29.545 \\
\hline 0.8 & 39.152 & 37.208 & 38.619 & 39.259 & 29.751 & 28.807 & 29.965 & 30.116 \\
\hline 0.9 & 39.877 & 37.926 & 39.336 & 39.971 & 30.306 & 29.245 & 30.490 & 30.645 \\
\hline 1 & 40.566 & 38.631 & 40.034 & 40.618 & 30.860 & 29.684 & 31.018 & 31.136 \\
\hline
\end{tabular}




\subsection{PERFORMANCE COMPRESSION RATIO}

Traditional compression methods usually fail to get a good quality image at high compression ratios. Fingerprint image 101_1.tif from FVC2000 DB1 database is taken as the reference image. The number of decomposition level in the transform is taken as 4. The Fig.4 and Fig.5 show the input image and the corresponding images after compression using wavelet, shuffled multiwavelet with existing prefilter, unshuffled multiwavelet with existing prefilter and unshuffled multiwavelet with optimum prefilter for the compression ratios 80:1, 100:1.

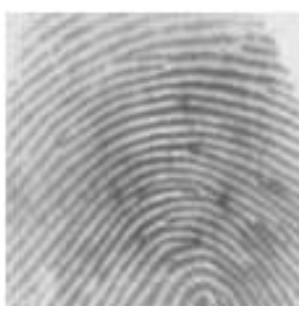

(a)

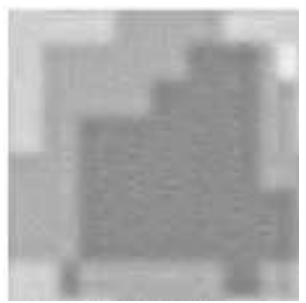

(c)

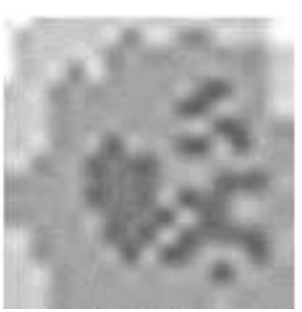

(b)

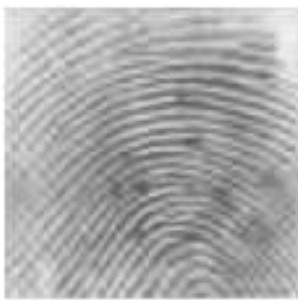

(d)

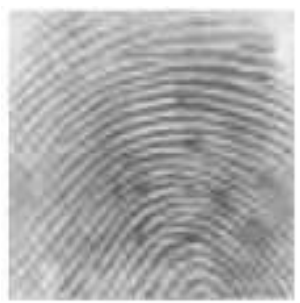

(e)

Fig.4. Compression at $\mathrm{CR}=80$ (a) input image, reconstructed images using (b) wavelet (PSNR=20.12dB) (c) shuffled multiwavelet (PSNR $=20.90 \mathrm{~dB}$ ) (d) unshuffled multiwavelet $(\mathrm{PSNR}=26.83 \mathrm{~dB})(\mathrm{e})$ unshuffled multiwavelet with optimum prefilter $(\mathrm{PSNR}=26.82 \mathrm{~dB})$

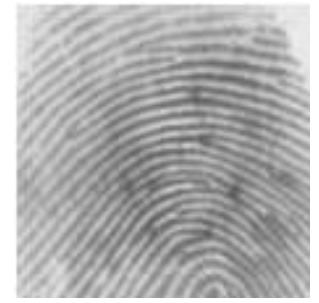

(a)

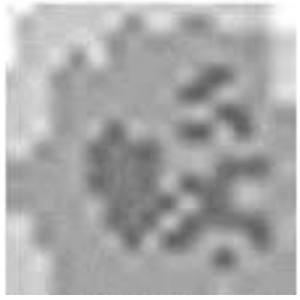

(b)

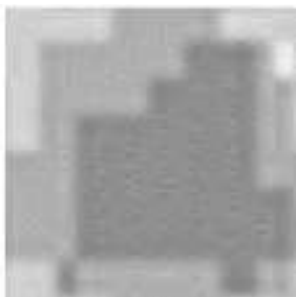

(c)

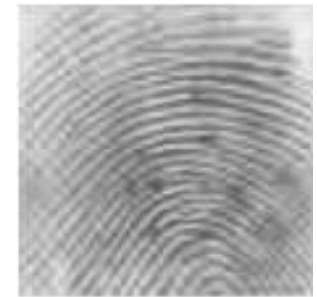

(d)

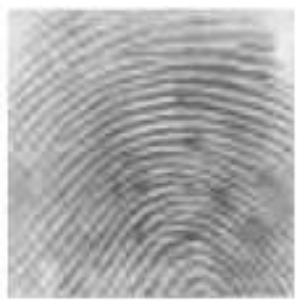

(e)

Fig.5. Compression at $\mathrm{CR}=100$ (a) input image, reconstructed images using (b) wavelet (PSNR=16.84dB) (c) shuffled multiwavelet $(\mathrm{PSNR}=19.29 \mathrm{~dB})$ (d) unshuffled multiwavelet $(\mathrm{PSNR}=25.70 \mathrm{~dB})(\mathrm{e})$ unshuffled multiwavelet with optimum prefilter $(\mathrm{PSNR}=25.65 \mathrm{~dB})$

At higher compression ratios like 80:1, 100:1 etc., wavelet based compression method as well as shuffled multiwavelet are not able to reconstruct the original image. Unshuffled multiwavelet with un-optimised prefilter based compression technique is giving an acceptable quality image at higher compression ratios compared to wavelets and other techniques. Unshuffled multiwavelet with optimised prefilter based compression techniques can be used for obtaining a better performance at low compression ratios. The Fig. 6 and Fig.7 show the input image and the corresponding images after compression using wavelet, shuffled multiwavelet with existing prefilter, unshuffled multiwavelet with existing prefilter and unshuffled multiwavelet with optimum prefilter for compression ratios 10:1 and $16: 1$.

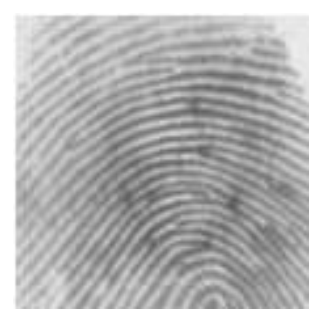

(a)

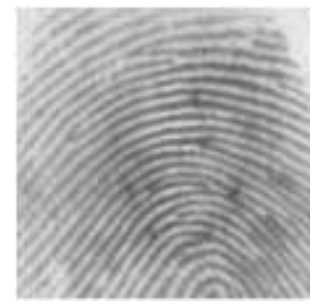

(c)

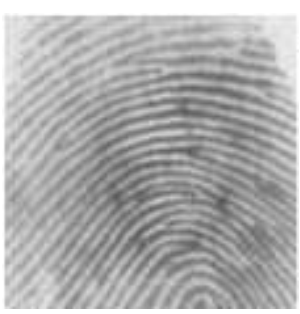

(b)

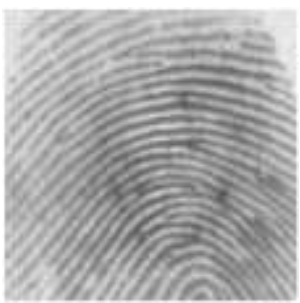

(d) 


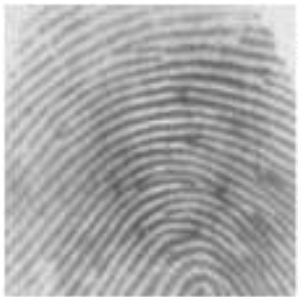

(e)

Fig.6. Compression at $\mathrm{CR}=10$ (a) input image, reconstructed images using (b) wavelet $(\mathrm{PSNR}=38.98 \mathrm{~dB})$ (c) shuffled multiwavelet (PSNR=37.48dB) (d) unshuffled multiwavelet $(\mathrm{PSNR}=38.49 \mathrm{~dB})(\mathrm{e})$ unshuffled multiwavelet with optimum prefilter $(\mathrm{PSNR}=39.31 \mathrm{~dB})$

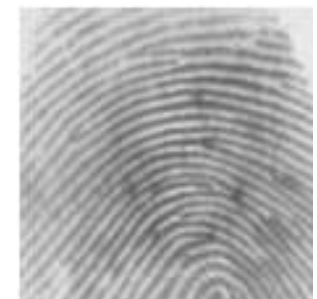

(a)

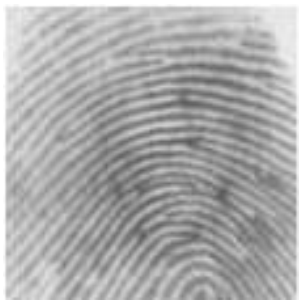

(c)

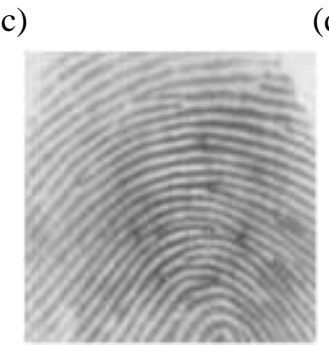

(e)

Fig.7. Compression at $\mathrm{CR}=16$ (a) input image, reconstructed images using (b) wavelet $(\mathrm{PSNR}=35.97 \mathrm{~dB})(\mathrm{c})$ shuffled multiwavelet (PSNR=34.19dB) (d) unshuffled multiwavelet $(\mathrm{PSNR}=35.99 \mathrm{~dB})$ (e) unshuffled multiwavelet with optimum prefilter $(\mathrm{PSNR}=36.42 \mathrm{~dB})$

Comparing Fig.4 to Fig.7, it can be observed that the proposed technique performs equally good at lower compression ratios and better at higher compression ratios compared to the wavelet and multiwavelet based techniques available in literature.

\section{CONCLUSION}

In this paper multiwavelet based fingerprint compression with optimised prefilter coefficients using SPIHT algorithm is proposed. SPIHT, the wavelet based compression algorithm offers a low quality image at lower bit rates due to lack of high frequency information. In order to get a better quality image at lower bit rates, unshuffled multiwavelet is used as transform. For getting an improved performance at higher bit rates, prefilter coefficients are optimised using a genetic algorithm. An improved performance for all the images in FVC 2000 DB1 database and FVC 2002 DB3 database is obtained and the corresponding average improvements in PSNR are $4.23 \mathrm{~dB} 2.52 \mathrm{~dB}$ respectively. The proposed technique using unshuffled multiwavelet with optimum prefilter gives better performance at all bit rates particularly at low bit rates compared to wavelet based compression techniques. So this technique can be used for fingerprint recognition at low bit rates.

\section{REFERENCES}

[1] V. Strela, P.N. Heller, G. Strang, P. Topiwala and C. Heil, "The Application of Multiwavelet Filterbanks to Image Processing", IEEE Transactions on Image Processing, Vol. 8, No. 4, pp. 548-563,1999.

[2] M. Ashok and D.T.B. Reddy, "Image Compression Techniques using Modified High Quality Multiwavelets", International Journal of Advanced Computer science and Applications, Vol. 2, No. 7, pp. 153-158, 2011.

[3] U.S. Ragupathy, D. Baskar and A. Tamilarasi, "New Method of Image Compression using Multiwavelets and Set Partitioning Algorithm", Proceedings of $10^{\text {th }}$ IEEE International Conference on Industrial and Information Systems, pp. 1-6, 2008.

[4] S. Radhakrishnan and J. Subramaniam, "Fingerprint Compression using Multiwavelets", International Journal of Signal Processing, Vol. 2, No. 2, pp. 78-87, 2006.

[5] X.G. Xia, J.S. Geronimo, D.P. Hardin and B.W. Suter, "Design of Prefilters for Discrete Multiwavelet Transforms", IEEE Transactions on Signal Processing, Vol. 44, No. 1, pp. 25-35, 1996.

[6] K. Attakitmongcol, D.P. Hardin and D.M. Wilkes, "Multiwavelet prefilters. II. Optimal orthogonal prefilters", IEEE Transactions on Image Processing, Vol. 10, No. 10, pp. 1476-1487, 2001.

[7] H. Shi, Y. Cai and Z. Qiu, "On Design of Multiwavelet Prefilters", Applied Mathematics and Computation, Vol. 172, No. 2, pp. 1175-1187, 2006.

[8] S. Esakkirajan, T. Veerakumar, V.S. Murugan and R. Sudhakar, "Fingerprint Compression using Contourlet Transform and Multistage Vector Quantization", International Journal of Biological and Medical Sciences, Vol. 1, No. 2, pp. 140-147, 2006.

[9] G. Shao, Y. Wu, A. Yong, X. Liu and T. Guo, "Fingerprint Compression based on Sparse Representation", IEEE Transactions on Image Processing, Vol. 23, No. 2, pp. 489$501,2014$.

[10] K.T. Shanavaz and P. Mythili, "Faster Techniques to Evolve Wavelet Coefficients for better Fingerprint Image Compression", International Journal of Electronics, Vol. 100, No. 5, pp. 655-668, 2013.

[11] B.S. Emmanuel, M.D. Muazu, S.M. Sani and S. Garba, "Improved Algorithm for Biometric Fingerprint Image 
Compression", American Journal of Computation, Communication and Control, Vol. 1, No. 5, pp. 75-85, 2014.

[12] H. Grailu, "Improving the Fingerprint Verification Performance of Set Partitioning Coders at Low Bit Rates", Multimedia Tools and Applications, Vol. 76, No. 7, pp. 9959-9991, 2017.

[13] M.B. Martin and A.E. Bell, "New Image Compression Techniques using Multiwavelets and Multiwavelet Packets", IEEE Transactions on Image Processing, Vol. 10, No. 4, pp. 500-510, 2001.

[14] R. Sumalatha and M.V. Subramanyam, "Medical Image Compression using Multiwavelets for Telemedicine Applications", International Journal of Scientific and Engineering Research, Vol. 2, No. 9, pp. 1-4, 2011.
[15] J.Y. Tham, L. Shen, S.L. Lee and H.H. Tan, "A General Approach for Analysis and Application of Discrete Multiwavelet Transforms", IEEE Transactions on Signal Processing, Vol. 48, No. 2, pp. 457-464, 2000.

[16] A. Said and W.A. Pearlman, "A New, Fast, and Efficient Image Codec based on Set partitioning in Hierarchical Trees", IEEE Transactions on Circuits and systems for Video Technology, Vol. 6, No. 3, pp. 243-250, 1996.

[17] M.D. Adams and R. Ward, "Wavelet Transforms in the JPEG-2000 Standard", Proceedings of IEEE Pacific Rim Conference on Communications, Computers and Signal Processing, Vol. 1, pp. 160-163, 2001. 